\title{
Design and Implementation of a Measurement-based Adaptive Wide-Area Damping Controller Considering Time Delays
}

\author{
Feifei Bai ${ }^{1}$, Lin Zhu ${ }^{2}$, Yilu Liu ${ }^{2,3}$, Xiaoru Wang ${ }^{1}$, Kai Sun ${ }^{2}$, Yiwei Ma ${ }^{2}$, Mahendra Patel ${ }^{4}$, Evangelos Farantatos ${ }^{4}$, and Navin \\ Bhatt $^{4}$ \\ *Corresponding author: lzhu12@utk.edu \\ ${ }^{1}$ Southwest Jiaotong University, Chengdu, China \\ ${ }^{2}$ University of Tennessee, Knoxville, USA \\ ${ }^{3}$ Oak Ridge National Laboratory, Oak Ridge, USA \\ ${ }^{4}$ Electric Power Research Institute, USA
}

\begin{abstract}
Wide-area measurement systems enable the wide-area damping controller (WADC) to use remote signals to enhance the small signal stability of large scale interconnected power systems. System operating condition variations and signal transmission time delays are the major factors to worsen the damping effect and even deteriorate the system stability. This paper proposes a novel measurement-based adaptive wide-area damping control scheme using oscillation mode prediction and system identification techniques. These techniques adjust the parameters of WADC as well as the time delay compensation in an online environment. To achieve fast online implementation, an identified high order multi-input multi-output (MIMO) model is deformed into a low order single-input single-output (SISO) model according to the residue of MIMO model. The SISO model can accurately represent the power system dynamics in the form of a transfer function, capturing the dominant oscillatory behaviors in the frequency range of interest. Moreover, the WADC has been implemented on a hardware test-bed (HTB) by adding its output signal to the excitation system of a selected generator. The effectiveness of the proposed measurement-based adaptive WADC has been demonstrated in a two-area four-machine system on the HTB under various disturbance scenarios.
\end{abstract}

Keywords -- adaptive wide-area damping control system, hardware test-bed, system identification, residue, time delay compensation, wide-area measurement system.

\section{INTRODUCTION}

With the increasing interconnection of large power grids, the power exchange among different areas via long distance transmission lines has significantly increased. The inter-area oscillations have been a critical issue limiting the power transfer capability and even deteriorating the security of the entire power system [1]-[2]. Therefore, damping of inter-area oscillations is one of the main concerns in the enhancement of power transmission and improving power system stability [3]. With the development of the wide-area measurement systems (WAMS), power system controllers can now utilize remote feedback signals from different locations of the power grid. The utilization of global signals can provide a better observation of inter-area modes, and overcome the shortcomings of local power system stabilizers (PSSs) [3]-[6]. However, most of wide-area damping controllers (WADCs) are tuned based on a number of typical operating conditions [3]-[7]. Although such methods are based on the exact model of power system and are well suited for off-line designing, the performances of designed WADCs may degrade if the actual operating condition is significantly different from what was considered in the offline design procedure. In some extreme cases, WADCs even provide negative damping.

The robust control technology is firstly utilized to solve the operating condition variation. In general, a robust oscillation damping controller is designed based on a detailed system model under a selected dominant operating 
condition with bounded model uncertainty [8],[10]. The variations of the operating condition are reflected in the additive or multiplicative uncertainty of the system model. However, the number of operating conditions taken into consideration is limited due to computational complexity and increased chances of infeasibility. Additionally, in a real power system, the number of operating conditions is undoubtedly more than hundreds of thousands. Thus it is difficult to find a feasible solution for a polytope to accommodate the numerous operating conditions [11].

The adaptive control technology is another approach to improve the adaptivity of the controller. This technology can adjust the controller parameters based on the online estimated system model, and therefore, can adapt to the continuous variations in operating conditions. The adaptive control approach is more and more attractive as these technologies rely solely on PMU measurements since WAMS is capable to provide real-time measurements of the power system state with satellite-triggered time stamp in time intervals down to $20 \mathrm{~ms}$ [12][14].

On the other hand, although WADCs provide a great potential to improve the damping of inter-area oscillation, the signal time delays, mainly introduced by the long distance transportation of feedback signal, will degrade the damping performance and may even cause instability of the closed-loop system [15]-[16]. Those time delays can typically vary from tens to hundreds milliseconds, depending on the routines of signal transportation, communication protocols, and network load [17]. WADC design considering time delay has been addressed, such as applying a nonlinear bang-bang control method to deal with time delays [18] and designing robust controllers to handle the time delay as a part of the system uncertainties [19]-[20]. Although these methods could be used to design WADCs for various time delays offline, complex treatment of time delays would not only increase the processing time but also reduce the practicability of widearea damping control online. Considering these disadvantages, a simple but practical local time delay compensator is designed to eliminate the influence of the signal transmission time delay.

This paper presents the design and implementation of an adaptive wide-area damping control system (WADCS) based on solely wide-area measurements: (1) fast Fourier transform (FFT) is employed to preselect remote signals in each area as the output signals of the transfer function model for identification; (2) the autoregressive exogenous model (ARX) identification technology [21]-[22] is adopted to identify the multi-input multi-output (MIMO) system model; (3) a control loop is selected based on the residue of the MIMO ARX model; (4) a SISO ARX prediction model is constructed according to the control loop for adjusting the WADC parameters online; (5) a practical time delay compensator is designed to eliminate the influence of the time delay; and (6) real-time implementation of the proposed WADC is applied on a two-area four-machine system on hardware test-bed (HTB), which is a converter based reconfigurable power grid emulator system serving as a platform for power system control methodology test and demonstration.

The remaining of this paper is organized as follows. The methodology of the adaptive WADC design is introduced in Section II. In Section III, the proposed WADC is validated by the case study in a two-area four-machine system on hardware test-bed (HTB). Discussion and future work are given in Section IV. Section V concludes this paper.

\section{Methodology of AdAPTIVE WADC Design Considering Time Delay}

The overall structure of an adaptive WADC design considering time delay is shown in Fig. 1(a). The WADC is designed to damp a critical inter-area oscillation mode by providing supplementary damping control signal for PSS as shown in Fig. 1(a). A classical lead-lag type WADC is considered. The time delay from the remote signals for the whole 
control loop is simplified as one single delay $d$ at the feedback loop and represented by an $e^{-s d}$ block in Fig. 1(b). An ARX model is used to predict the feedback signal and obtain the oscillation for adjusting the controller parameters.

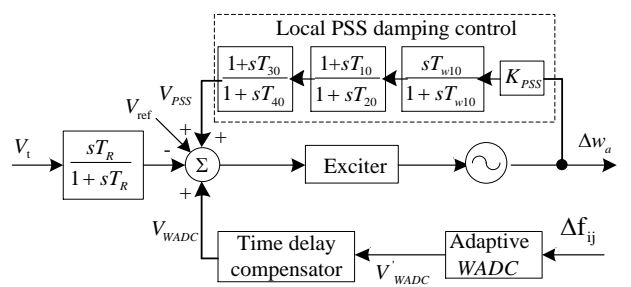

Fig. 1 (a) Supplementary damping control of PSS

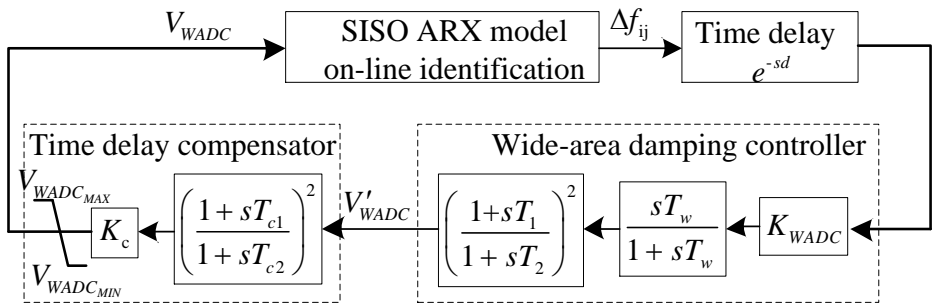

Fig. 1(b) Adaptive WADC considering time delay

As shown in Fig. 1(b), the transfer function of a classical WADC [3] is

$$
H_{W A D C}(\mathrm{~s})=K_{W A D C} \frac{T_{w} \mathrm{~s}}{1+T_{w} \mathrm{~s}}\left(\frac{1+s T_{1}}{1+s T_{2}}\right)^{\mathrm{m}}=K_{W A D C} H_{W A D C}^{\prime}(\mathrm{s})
$$

where $T_{1}$ and $T_{2}$ are the lead and lag time constants, respectively, $T_{\mathrm{w}}$ is the washout constant usually as $5-10 \mathrm{~s}, \mathrm{~K}_{\mathrm{WADC}}$ is the gain of the WADC, $m$ is the number of the lead-lag block and $m$ is usually given the value of 2 [4].

The details of the proposed adaptive WADCS design are described in the following subsections.

\section{A. Signal Detrending}

Trend in a time series is a slow, gradual change in some property of the series over the whole study time window. For system identification, all signals should be detrended. There are different detrending methods, e.g., first differencing, curve fitting and digital fitting. First differencing is used in this paper for practical application. It is defined as

$$
y(t)=y^{\prime}(t)-y^{\prime}(t-h)
$$

where $y^{\prime}(t)$ is the orginal measurement signal, $y(t)$ is the detrended signal, and $h$ is the sample time interval.

For dynamics study, we focus on the dynamics of the original signal $y^{\prime}(t)$ instead of the detrended signal $y(t)$. To recover the original signal from the detrended signal, the inverse form of first differencing filter is

$$
y^{\prime}(t)=y(t)+y^{\prime}(t-h)
$$

\section{B. MIMO ARX Model identification}

Fast online identification of the system model to capture all critical modes of the power system is the prerequisite of the adaptive oscillation damping control. Two categories of measurement-based models can be used for system identification: state-space model [25]-[29], and transfer function model [30]-[33]. The state-space representation is concerned not only with input and output properties of the system but also with its complete internal behavior. In contrast, the transfer function representation is concerned with and specifies only the input/output behavior [1]. Hence, the transfer function model identification can be an alternative to overcome the drawback of high computation burden of state-space methods. The linear MIMO ARX model is adopted to construct the system model off-line both to determine the control loop for each critical mode and to deform the SISO ARX model for each critical mode by predicting the future mode ahead for adjusting the controller parameters.

With measured signal $y(t)$ as the model output signal, measured signals $u(t)$ as the model input signal, the mathematical structure of the single-input single-output (SISO) ARX model structure [21] is described as: 


$$
y(t)+a_{1} y(t-1)+\cdots+a_{n_{a}} y\left(t-n_{a}\right)=b_{0} u(t)+b_{1} u(t-1)+\cdots+b_{n_{b}} u\left(t-n_{b}\right)+e(t)
$$

where $t$ is the time index, and $e(t)$ is a white noise. $n_{a}$ and $n_{b}$ are the orders of the signal $y(t)$ and $u(t)$, respectively.

With the SISO ARX model structure (4) the multi-input single-output (MISO) ARX model structure can be derived:

$$
y_{i}(t)+a_{i 1} y_{\mathrm{i}}(t-1)+\cdots+a_{i n_{a}} y_{i}\left(t-n_{a i}\right)=\sum_{j=1}^{M}\left(b_{i j 0} u_{j}(t)+b_{i j 1} u_{j}(t-1)+\cdots+b_{i j n_{b i j}} u_{j}\left(t-n_{b i j}\right)\right)+e(t)
$$

For the simplification, (5) can be further expressed in vector form as:

$$
\mathbf{a}_{i} \mathbf{y}_{i}(t)=\sum_{j=1}^{M} \mathbf{b}_{i j} \mathbf{u}_{j}(t)+\mathrm{e}_{\mathrm{i}}(t)
$$

where

$\mathbf{a}_{i}=\left[1, a_{i 1}, \cdots, a_{i n_{a}}\right], \mathbf{y}_{i}(t)=\left[\mathrm{y}_{i}(t), \mathrm{y}_{i}(t-1), \cdots, \mathrm{y}_{i}\left(t-n_{a i}\right)\right], \mathbf{b}_{i j}=\left[b_{i j 0}, b_{i j 1}, \cdots, b_{i j n_{a i j}}\right], \mathbf{u}_{j}(t)=\left[u_{j}(t), \cdots, u_{j}\left(t-n_{b i j}\right)\right]$.

Based on (6), the multi-input multi-output (MIMO) ARX model structure can be obtained as follows:

$$
\left.\begin{array}{c}
\mathbf{a}_{1} \mathbf{y}_{1}(t)=\sum_{j=1}^{M} \mathbf{b}_{1 j} \mathbf{u}(t)+\mathrm{e}_{1}(t) \\
\vdots \\
\mathbf{a}_{N} \mathbf{y}_{N}(t)=\sum_{j=1}^{M} \mathbf{b}_{N j} \mathbf{u}(t)+\mathrm{e}_{1}(t)
\end{array}\right\}
$$

Therefore, the MIMO ARX model can be written as:

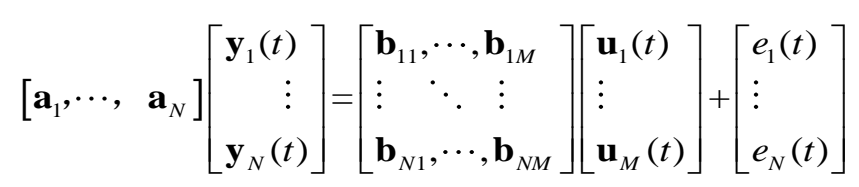

Since the equation (8) is linear, the parameters of the ARX model can be estimated by solving with linear least-square (LS) estimation method [21]. To evaluate the identified ARX model, a model fitness criterion is employed as the model accuracy index:

$$
F=\left(1-\sqrt{(\hat{Y}-Y)^{2}} / \sqrt{(\hat{Y}-\bar{Y})^{2}}\right) \times 100
$$

where $Y, \hat{Y}$, and $\bar{Y}$ are the estimated response, the measured response, and the mean value of the measured response, respectively. This index is used to reflect the accuracy of the model in describing system dynamics. A fitness of 100 means a perfect fit between the estimated response and the measured response, while a fitness of zero means the estimated response is no better than the mean value of the measured response.

In (8), the random part $e(t)$ is regarded as the error part of the identification. Assuming the identification is accurate, for simplicity, (8) can be written as:

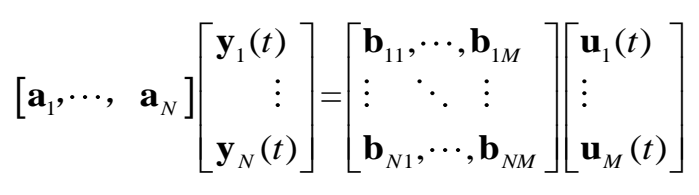

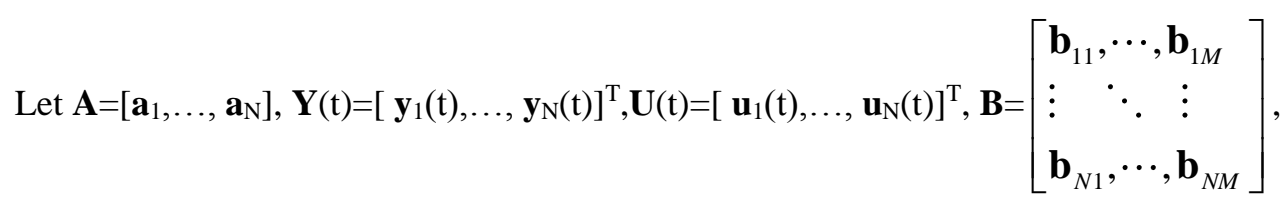

thus

$$
\mathbf{A Y}(\mathrm{t})=\mathbf{B U}(\mathrm{t}), \mathbf{Y}(\mathrm{t})=(\mathbf{B} / \mathbf{A}) \mathbf{U}(\mathrm{t})
$$

The MIMO ARX model in (10) is a transfer function matrix about the power system input $\mathbf{U}(\mathbf{t})$ and output $\mathbf{Y}(\mathbf{t})$ in the discrete-time domain. If converted into the continuous-time domain, the system transfer function can be represented as: 


$$
\mathbf{G}(\mathrm{s})=\mathbf{Y}(\mathrm{s}) \mathbf{U}^{-1}(\mathrm{~s})=\left[\mathbf{A}^{-1}(\mathrm{z}) \mathbf{B}(\mathrm{z})\right]_{z=e^{s T_{s}}}
$$

where $T_{s}$ is the sampling period. $s$ and $z$ are the continuous-time domain and the discrete-time domain sign, respectively.

Now, $\mathrm{G}(\mathrm{s})$ can be expanded in partial fractions as:

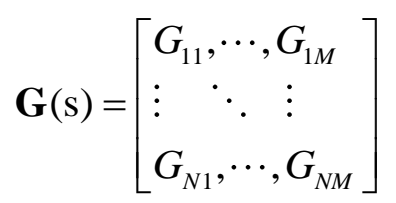

where

$$
\mathbf{G}_{N M}(s)=\frac{R_{N M 1}^{\prime}}{s-p_{N M 1}}+\cdots+\frac{R_{N M n 1}^{\prime}}{s-p_{N M n 1}}
$$

where $R_{N M n 1}^{\prime}$ is the residue of $G_{M N}(s)$ at pole $p_{N M n 1}$.

Since the problem focuses on small signal stability control, an identification routine is used to accurately estimate linearized models of the power system, capturing the critical dynamics in the frequency range of interest, i.e., 0.1-1.0 Hz. Thus, (14) can be simplified as:

$$
G_{N M}(s)=\frac{R_{N M 1}}{s-\lambda_{N M 1}}+\cdots+\frac{R_{\mathrm{NMk}}}{s-\lambda_{N M k}}+\cdots+\frac{R_{N M n 2}}{s-\lambda_{N M n 2}}
$$

where $R_{N M k}$ is the residue of $G(s)$ at the eigenvalue $\lambda_{N M k}, \mathrm{NM} n_{2}<\mathrm{NM} n_{1}$.

\section{SISO ARX Model Identification}

Obtaining the MIMO model is the initial design step to get the residues of all input-output pairs in the critical range of oscillation mode. However, the MIMO model is difficult to be implemented to an online adaptive control because of the computational burden and convergence time on the identification stage. The second design stage deforms the centralized MIMO model into low order decentralized SISO models.

Assuming the critical mode is $\lambda_{N M k}$, the largest residue for all the transfer functions in the transfer function matrix is $\mathrm{R}_{\mathrm{NMk}}$ $\mathrm{y}_{\mathrm{Ni}}$ and $\mathrm{y}_{\mathrm{Nj}}$ are the best observation signals in two areas corresponding to the most critical inter-area mode, then the input signal $u_{\mathrm{M}}$ and the output deviation signal $y_{N i-N j}$ corresponding to this transfer function are selected as the input and output signals to construct the SISO ARX model for the online prediction.

$$
y_{N i-N j}(t)=c_{1} y_{N i-N j}(t-h)+\cdots+c_{n_{c}} y_{N i-N j}\left(t-n_{c} h\right)+d_{1} u(t-h)+\cdots+d_{n_{d}} u\left(t-n_{d} h\right)+e(t)
$$

(16) can be written as

$$
y_{N i-N j}(t)=\mathrm{C}(q) \mathrm{y}_{N i-N j}(t-h)+\mathrm{D}(q) u(t-h)+e(t)
$$

where $y_{N i-N j}(t)=y_{N i}(t)-y_{N j}(t), \mathrm{C}(\mathrm{q})=c_{1} q^{-1}+\cdots+c_{n_{c}} q^{-n_{c}}, \mathrm{D}(\mathrm{q})=d_{1} q^{-1}+\cdots+d_{n_{d}} q^{-n_{d}}$.

where $q^{-1}$ is a backward shift operator and $q^{-1} y(t)=y(t-1) . h$ is the sample time interval. $y_{N i-N j}(t)$ is the prediction of the feedback signal, $e(t)$ is the estimation error. The parameters of the model can be estimated by recursive least square estimation method [21].

The reduction to be a SISO model can reduce the computational burden and hence improve the convergence time without losing much of the relevant information in the frequency range of interest, i.e., 0.1 1Hz. To ensure the SISO model can capture dominate dynamic characteristics of the system, time domain and frequency domain verification will be carried out. 


\section{Selection of the Remote Feedback Signal and PSS Location}

FFT is adopted to preselect the best feedback signal in each area by extracting the measurement signals from time domain to frequency domain [34]-[35]. The measurement signals are ranked in descending order according to the amplitude in the frequency ranges of the selected modes. Then the highest signal in each area will be selected as the variables to construct the MIMO ARX model. Moreover, the difference between the two feedback signals in the two oscillating areas is selected as the output signal of the SISO model, which will be used to predict the dominate mode.

The residue method is used to select the location of PSS supplementary control. The open-loop transfer function is obtained by (13) and (15) in Section II-B. The residue $\mathrm{R}_{\mathrm{NMk}}$ is associated with a critical mode $\lambda_{N M k}$. The supplementary control signal of PSS is located at the machine that has the largest residue to give the largest change of $\lambda_{N M k}$ [3]. In general, the residues are complex numbers. The best one is given by the maximum value of the residue magnitude.

\section{E. WADC Design}

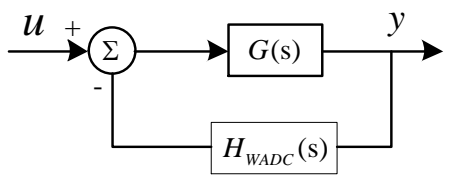

Fig. 2. Insertion of a PSS in the system

For each decentralized controller as shown in Fig. 2, the eigenvalues of the new system are the roots of the following characteristic function

$$
1+H_{W A D C}(\mathrm{~s}) \mathrm{G}_{N M}(\mathrm{~s})=1+H_{W A D C}(\mathrm{~s})\left(\frac{R_{N M 1}}{s-\lambda_{N M 1}}+\cdots+\frac{R_{\mathrm{NMk}}}{s-\lambda_{N M k}}+\cdots+\frac{R_{N M n 2}}{s-\lambda_{N M n 2}}\right)=0
$$

If the change introduced to $\lambda_{\mathrm{NMk}}$ is small, the change in $\lambda_{\mathrm{NMk}}$ can be approximated as

$$
\Delta \lambda_{N M \mathrm{k}}=R_{\lambda_{N M \mathrm{k}}} H_{W A D C}\left(\lambda_{N M \mathrm{k}}\right)
$$

In order to achieve the damping of one oscillation mode without changing its oscillation frequency, $\Delta \lambda_{N M k}$ should be a negative real number so as to shift $\lambda_{\text {NMK }}$ horizontally to the left-half of the complex plane, that is

$$
\Phi_{N M k}=\arg \left(H_{\text {WADC }}\left(\lambda_{N M k}\right)\right)=180-\arg \left(R_{N M k}\right)
$$

where $\Phi_{N M k}$ is the compensation angle for oscillation mode $\lambda_{N M k}$.

Once the desired damping is fixed, the desired position of the eigenvalue $\lambda_{\text {Ides }}$ is determined, then

$$
K_{W A D C}=\left|\frac{\Delta \lambda_{N M \mathrm{k}}}{R_{\lambda_{N M \mathrm{k}}} H_{W A D C}^{\prime}\left(\lambda_{N M \mathrm{k}}\right)}\right|=\left|\frac{\lambda_{\text {ides }}-\lambda_{N M \mathrm{k}}}{R_{\lambda_{N M \mathrm{k}}} H_{W A D C}^{\prime}\left(\lambda_{N M \mathrm{k}}\right)}\right|
$$

Other parameters can be calculated as[4]

$$
T_{1}=\frac{1}{\omega \sqrt{\alpha}}, T_{2}=\alpha T_{1}, \alpha=\frac{1-\sin \frac{\phi_{N M \mathrm{k}}}{2}}{1+\sin \frac{\phi_{N M \mathrm{k}}}{2}}, w=2 \pi f_{N M k}
$$

where $f_{\mathrm{MNk}}$ is the oscillation frequency of the mode $\lambda_{\text {NMk }}$. 


\section{F. Signal Time Delay Compensation}

Accurate time from the global positioning system (GPS) would be received locally in both the PMUs and the time delay compensator. Wide-area phasors measured by the PMUs at time $t_{1}$ are collected and resynchronized by the PDC and in turn processed by the adaptive WADC to generate the wide-area damping control signal V'WADC with a time stamp [ $\left.t_{1}\right]$. When the local time delay compensator, which is installed close to the generator exciter, receives the control signal V'WADC $\left[t_{2}\right]$, the signal will be re-labelled with a new time stamp [ $\left.t_{2}\right]$ freshly obtained from GPS and the exact time delay can be calculated accurately as $\tau=t_{2}-t_{1}$ because of the high-resolution time service provided by the GPS.

Once the time delay is derived from the time stamps, it can be modeled and compensated as shown in Fig. 3.

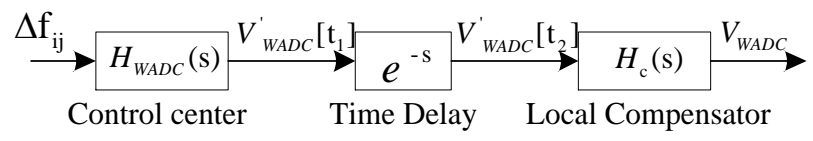

Fig.3 Time delay Compensation

If the most critical inter-area mode is $\lambda$, the signal can be expressed as:

$$
f(t)=A e^{\sigma t} \cos (\theta+w t)
$$

where $\mathrm{A}$ is the initial amplitude, $\sigma$ is the decay factor, $\theta$ is the initial phase, $\omega$ is the angular frequency.

If the time delay is $\tau$, the signal will be:

$$
f(t+\tau)=A e^{\sigma(t+\tau)} \cos (\theta+w(t+\tau))
$$

This means that the signal time delay effects on the displacement of $\lambda$ can be decomposed into gain drift $\gamma$ and the phase lag $\Phi$, which will influence the damping of the mode.

$$
\Phi=w \tau, \quad \gamma=\mathrm{e}^{-\sigma \tau}
$$

In order to eliminate the effects of the time delay, the following transfer function will be used to compensate the phase lead/lag and the gain drift:

$$
H_{\mathrm{c}}(\mathrm{s})=K_{\mathrm{c}}\left(\frac{1+s T_{\mathrm{cl}}}{1+s T_{c 2}}\right)^{2}
$$

where $T_{\mathrm{c} 1}=\frac{1}{\omega \sqrt{\alpha_{\mathrm{c}}}}, T_{\mathrm{c} 2}=\alpha_{\mathrm{c}} T_{\mathrm{c} 1}, \alpha_{\mathrm{c}}=\frac{1-\sin \frac{w \tau}{2}}{1+\sin \frac{w \tau}{2}}, K_{\mathrm{c}}=\beta \frac{1}{\gamma}, 0<\beta<1$.

where $\beta$ can be adjusted according to the performance of the compensation. The parameters of the transfer function will be tuned using the same approach for WADC parameter tuning approach.

\section{G. Summary of the Proposed Adaptive Control Steps}

Fig. 4 shows the proposed adaptive wide-area damping control scheme with online system identification consideration of time delays. The adaptive wide-area damping control scheme mainly consists of 1) the offline MIMO model construction using the historical data, 2) control signal selection according to the residue of the MIMO model, 3) SISO prediction model identification and 4) WADC and time delay parameters update according to the predicted dominant mode by the SISO model. The controller algorithm operates on the sampled time interval $h$ and can be triggered by events when the dynamic response is over the threshold $\left[\Delta \mathrm{f}_{\mathrm{ijmin}}, \Delta \mathrm{f}_{\mathrm{ijmax}}\right]$ as shown in Fig. 1(b). 


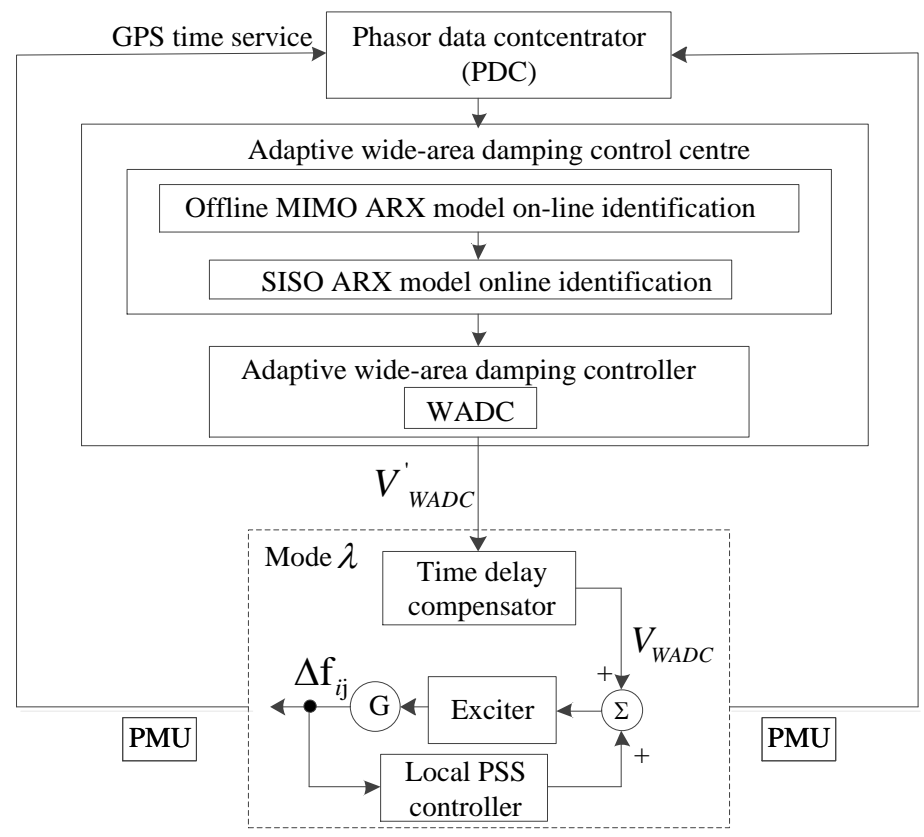

Fig. 4. Measurement-based Adaptive wide-area damping control scheme with consideration of signal time delay

The following are the major steps to realize the wide-area damping control scheme:

Step 1) Detrend the measurement signals.

Step 2) Select 20 seconds bus frequency signals after the first swing of the dynamic responses. Apply the FFT analysis method to preselect the best feedback signals in each area within the oscillation frequency range 0.1-1.0Hz.The selected signals will be the output of the MIMO ARX model.

Step 3) Choose all the voltage signals for 20 seconds of the excitation systems installed PSS as the input of the MIMO ARX model.

Step 4) Construct the MIMO ARX model with the selected signals selected by Step 2) and Step 3). Residues are obtained to decide the best control signal for each critical mode.

Step 5) Construct the SISO ARX model. According to the largest residue of each critical mode obtained from the MIMO model, the input and output signal pair corresponding to the residue is selected as the input and output of the SISO model, which is used to do the online prediction for each decentralized WADC. In order to obtain the order and initialized parameters of the SISO model, one event data is used to train this SISO model. Once the structure of the SISO model is obtained, the parameters of the SISO model will be updated using the recursive least square approach provided in [21].

Step 6) Initialize the parameters of WADC and time delay compensation model with the offline identified mode and residue at $t=0$.

Step 7) Update the parameters of the WADC according to the predicted mode and residue by the SISO model and produce the WADC output V' WADC at [ $\left.t_{1}\right]$, which will be sent to the generator selected output location of Step 5).

Step 8) V'WADC is measured locally at $\left[t_{2}\right]$ with the help of the GPS time service. The time delay is $\left[t_{2}-t_{1}\right]$, thus the parameters of the time delay compensation model can be updated to produce the control signal VWADC to suppress the dominate mode.

Step 9) Go back to Step 7) to start a new cycle of adaptive wide-area damping control when the dynamic response is over the threshold $\left[\Delta \mathrm{f}_{\mathrm{ijmin}}, \Delta \mathrm{f}_{\mathrm{ijmax}}\right]$. 


\section{CASE STUDY}

A large scale power system is a complex network with thousands of buses, hundreds of generators and many interactions between multiple areas with several inter-area oscillation modes. Moreover, each inter-area mode involvs a large number of generators. Owing to the complexity and expensive nature, it is impractical to demonstrate the effectiveness of the new control strategy in a real power grid. Hence, the proposed adaptive WADC is implemented in a two-area four-machine system [36] on a real-time power grid emulation platform to show its effectiveness. Although the existing platform only has one inter-area mode, the concept is applicable to any adaptive hierarchical inter-area damping control.

\section{A. Hardware Test-bed (HTB) Platform}

The HTB [36] in the National Science Foundation and Department of Energy (NSF/DOE) engineering research centerCenter for Ultra-wide-area Resilient Electric Energy Transmission Networks (CURENT), USA, is a platform built for power grid control methodology test and demonstration. The two-area four-machine system as shown in Fig.5 is now emulated on the HTB [37], which provides a perfect environment for WADC implementation, testing, and demonstration. The configuration of the HTB is shown in Fig. 6. A LabVIEW-based control system has been developed to emulate some functions of an actual power system control center. It gathers the measurement data from monitoring devices in the HTB and sends control commands to virtual generators through Ethernet. MATLAB code of an adaptive WADC was integrated into the HTB control software using standard LabVIEW interface. The configuration and parameters of this two-area fourmachine system are described in [36].

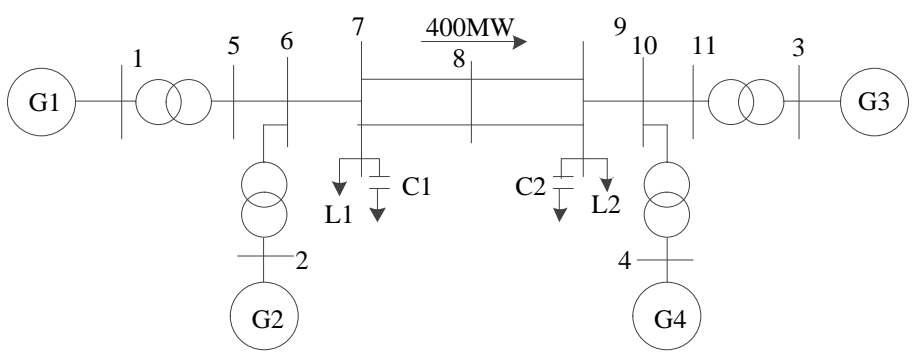

Fig. 5. Two-area four-machine system.

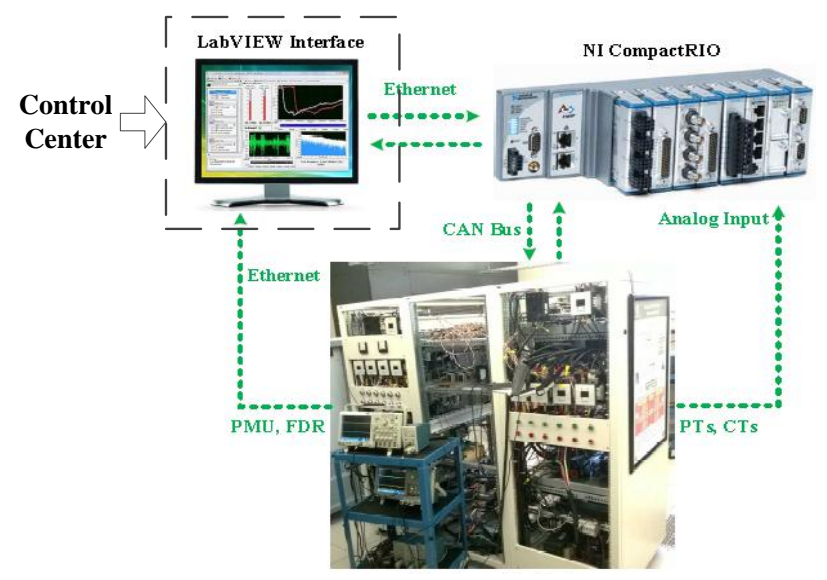

Fig. 6. HTB configuration.

All PMU equipped generators are monitored with frequency deviation measured and transmitted to the wide-area damping control center via the PMU data collection system. In case low-frequency oscillation arises, the local PSSs in 
generator 1 and generator 3 will act to suppress local modes while inter-area mode will be detected and suppressed by the WADC. The main suppression process is that (i) deforms the MIMO system model to the SISO prediction model according to the dominate mode; and (ii) tunes the WADC parameters online, sends the output signal V'WADC to the most participated generator and compensates the time delay to obtain the final control signal $\mathrm{V}_{\mathrm{WADC}}$.

\section{B. Model Construction and Verification}

Since there are only two PSSs in this system, the inputs of the MIMO model are the voltages of the excitation system on G1 and G3. While the output of the MIMO model should have a good observability to the dominant mode of the system, FFT analysis is applied to select the best feedback signal in each area as shown in Fig. 7.

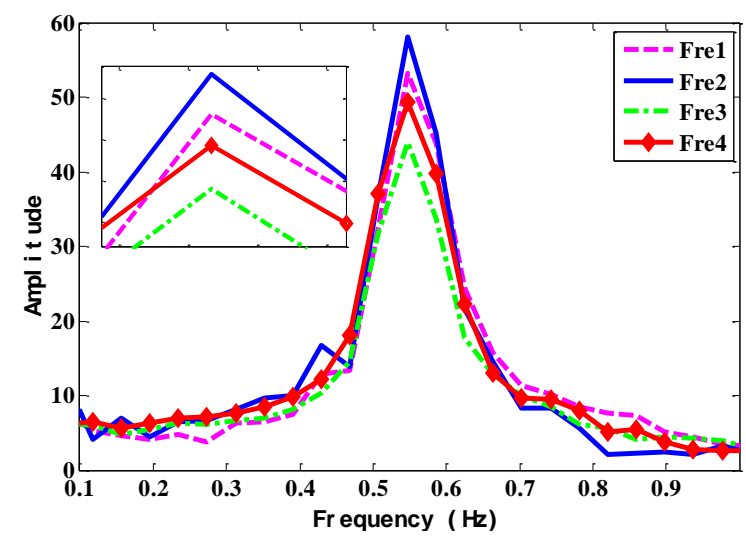

(a) Generation change

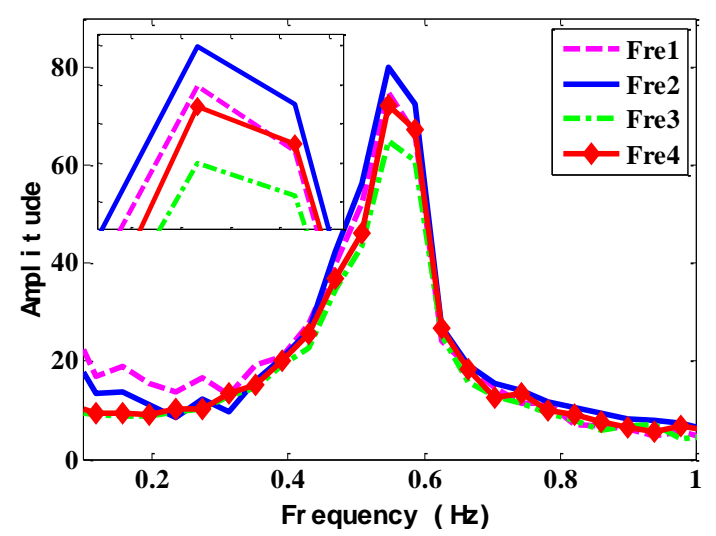

(b) Load change

Fig.7 FFT analysis to select the output of the model

As shown in Fig.7, bus frequency on G2 (Fre2) and G4 (Fre4) are the best observation signals in each area. Therefore Fre2 and Fre4 are selected as the output signals of the MIMO model. The MIMO model is then constructed as a two-input two-output ARX model.

The small signal stability characteristics of the experimental system are analyzed by the measurement data after a generation increase from $0.3 p u$ to $0.7 \mathrm{pu}$. The mode are shown in Table I. The inter-area mode at $0.58 \mathrm{~Hz}$ is the dominant mode of the system, which is selected as the target mode for WADC design.

\section{TABLE I}

MODAL ANALYSIS OF THE EXPERIMENTAL SYSTEM

\begin{tabular}{c|c|c}
\hline Mode & Frequency $(\mathrm{Hz})$ & Damping Ratio (\%) \\
\hline Inter-area & 0.58 & 5.32 \\
\hline Local & 1.57 & 13.98 \\
\hline Local & 1.14 & 17.17 \\
\hline
\end{tabular}

The residue of the MIMO transfer function model at the dominant mode are shown in Table II and the largest residue is considered as one. The others are normalized. Residues measure the ability to move the eigenvalue of the target mode to the left plane, so the larger the value, the better. Therefore, the signal Vex_1 on generator Bus 1 was selected as the optimal actuation signal to control the dominant mode. Considering the signal difference between two oscillating areas having better observability to the inter-area mode than single signal in each area, the frequency difference Fre 2-4 between 
G 2 and G 4 is selected as the feedback signal to construct the SISO prediction model. The model construction process is described in Fig. 8. The SISO model is verified in frequency and time domain as shown in Table III and Fig. 8.

TABLE II

CONTROl Signal SELECTION By REsidue CALCUlATION

\begin{tabular}{c|c|c}
\hline Input & Exciter & Residue \\
\hline 1 & 1 & 1 \\
\hline 2 & 3 & 0.9 \\
\hline
\end{tabular}

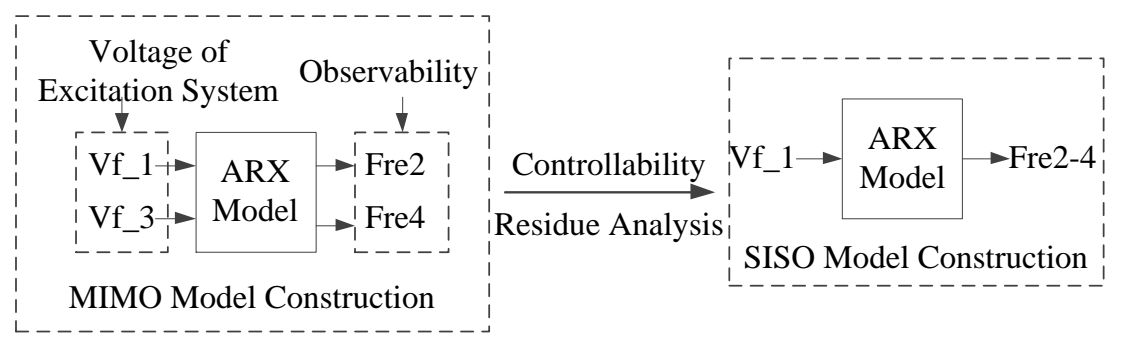

Fig.8 Model construction process

TABLE III

MODAL ANALYSIS OF THE EXPERIMENTAL SYSTEM

\begin{tabular}{c|c|c}
\hline Mode & Frequency(Hz) & Damping Ratio (\%) \\
\hline Inter-area & 0.58 & 3.8 \\
\hline
\end{tabular}

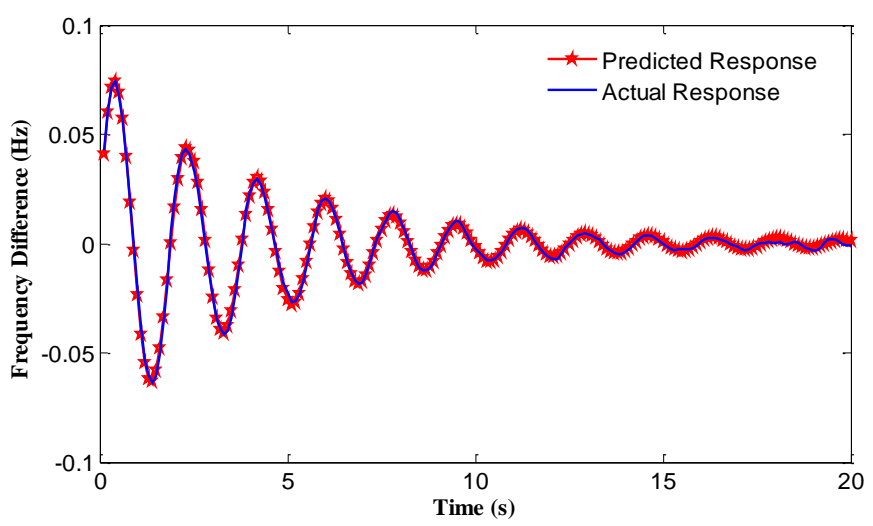

Fig.9 SISO model validation in time domain

The identified model was verified in frequency domain and time domain, which are shown in Table I and Fig.9. The reduction result shows that the SISO model can capture the dominate mode and can predict the frequency response accurately.

\section{WADC and Time Delay Compensation Design}

The initialization parameters of control system to damp the dominant mode are shown in Table IV. In order to analyze the effectiveness of the WADC and the time delay compensation model, the root loci of the system in three scenarios are analyzed as shown in Fig.10. As we can see from the figure, if there is no time delay compensation, a little gain change of the WADC will change the frequency of the dominate mode a lot while the damping ratio only increase a little bit. On the contrary, if the proper time delay compensation added into the WADC, the control performance is similar or better to the 
WADC when there is no time delay in the system. Therefore, the proposed control scheme can be implemented to the real-time HTB system with updated parameter along with the mode change during different operation conditions.

TABLE IV

PARAMETERS OF CONTROLLER AND TIME DELAY COMPENSATION

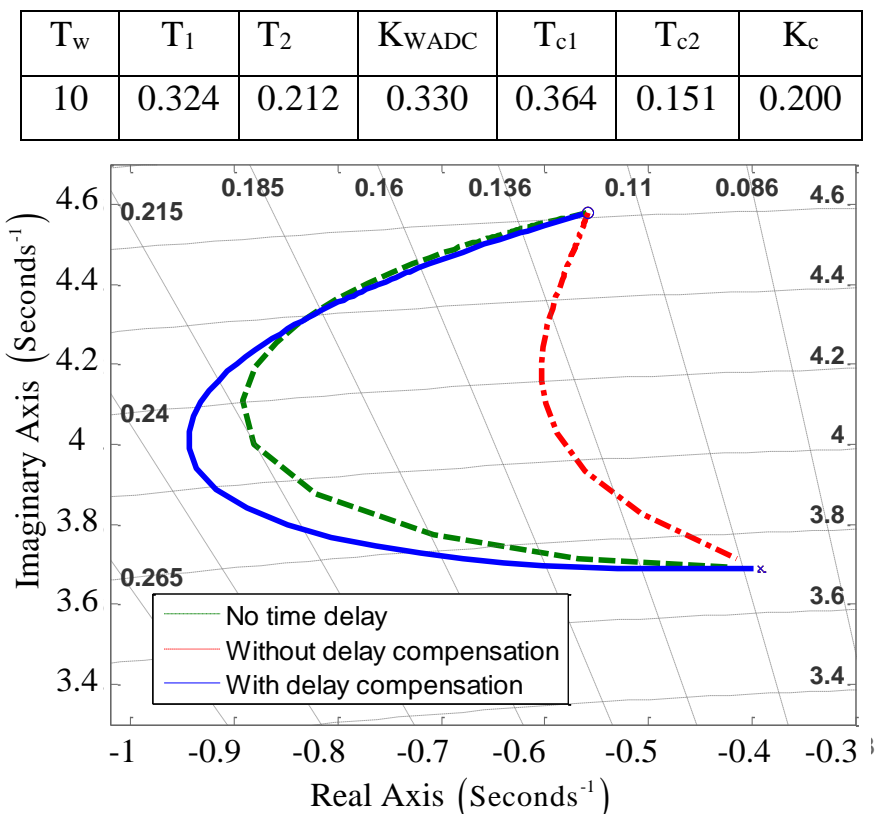

Fig.10 Root locus analysis in three scenarios

\section{Control Performance}

In order to test the effectiveness and robustness of the proposed adaptive WADCS, the following four typical scenarios and the captured dominate mode during different operations were shown in Table V. Parameters for adaptive WADC and time delay compensation used in each case are listed in Table VI. In addition, the time delay in the communication channel of HTB is $200 \mathrm{~ms}$.

TABLE V

CASE STUdy DetaILS

\begin{tabular}{c|c|c|c|c|c|c}
\hline Case & Installed PSS & Event Type & Event Location & Change (pu) & Frequency(Hz) & Damping Ratio \\
\hline 1 & G1,G3 & Load Increase & L2 & 0.4 to 0.7 & 0.58 & $4.2 \%$ \\
\hline 2 & G1,G3 & Generation Increase & G1 & 0.3 to 0.7 & 0.56 & $4.8 \%$ \\
\hline 3 & G1 & Load Increase & L1 & 0.4 to 1.0 & 0.56 & $0.38 \%$ \\
\hline 4 & G1 & Generation Trip & G3 & 0.4 to 0.05 & 0.65 & $1.67 \%$ \\
\hline
\end{tabular}

TABLE VI

PARAMETERS OF ADAPTIVE WADC AND TIME DELAY COMPENSATION

\begin{tabular}{|c|c|c|c|c|c|c|c|}
\hline Case No. & $\mathrm{T}_{\mathrm{w}}$ & $\mathrm{T}_{1}$ & $\mathrm{~T}_{2}$ & $\mathrm{~K}_{\mathrm{WADC}}$ & $\mathrm{Tc} 1$ & $\mathrm{Tc} 2$ & $\mathrm{Kc}$ \\
\hline Case 1 & 10 & 0.3624 & 0.2077 & 0.322 & 0.3984 & 0.1890 & 0.2062 \\
\hline Case 2 & 10 & 0.3324 & 0.2430 & 0.331 & 0.4071 & 0.1984 & 0.2069 \\
\hline Case 3 & 10 & 0.4408 & 0.1832 & 0.329 & 0.4071 & 0.1984 & 0.2005 \\
\hline Case 4 & 10 & 0.2889 & 0.2076 & 0.328 & 0.3728 & 0.1608 & 0.2027 \\
\hline
\end{tabular}




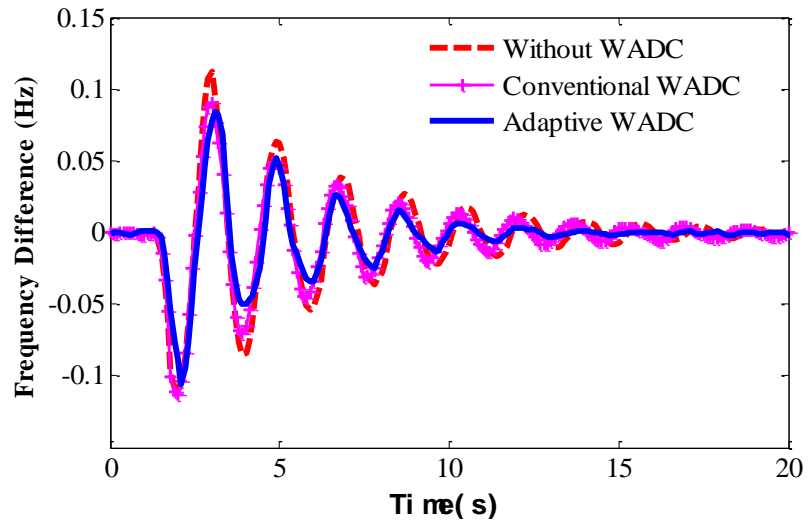

(a) Control performance comparison of Case 1

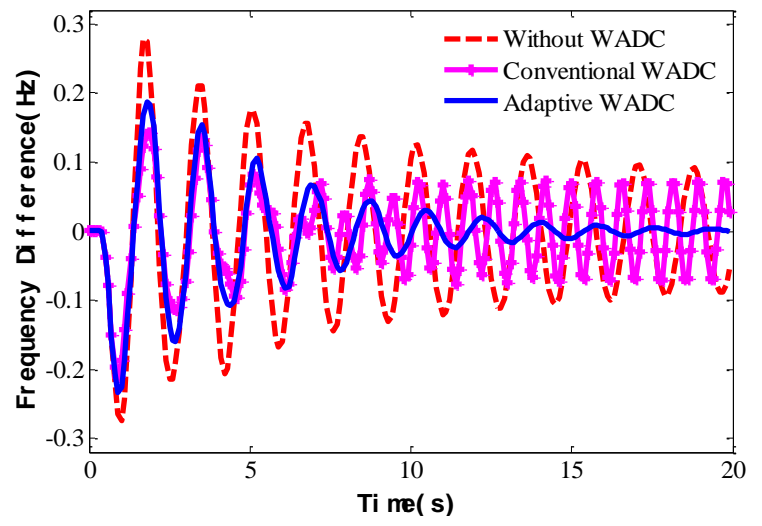

(c) Control performance comparison of Case 3

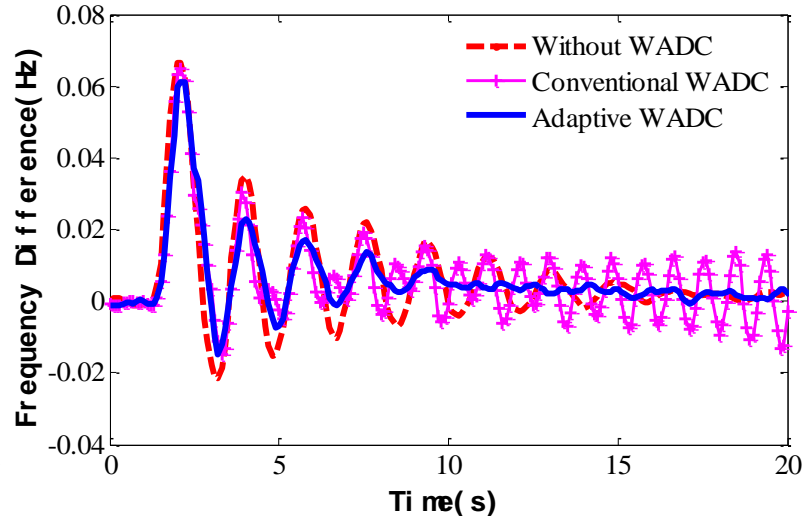

(b) Control performance comparison of Case 2

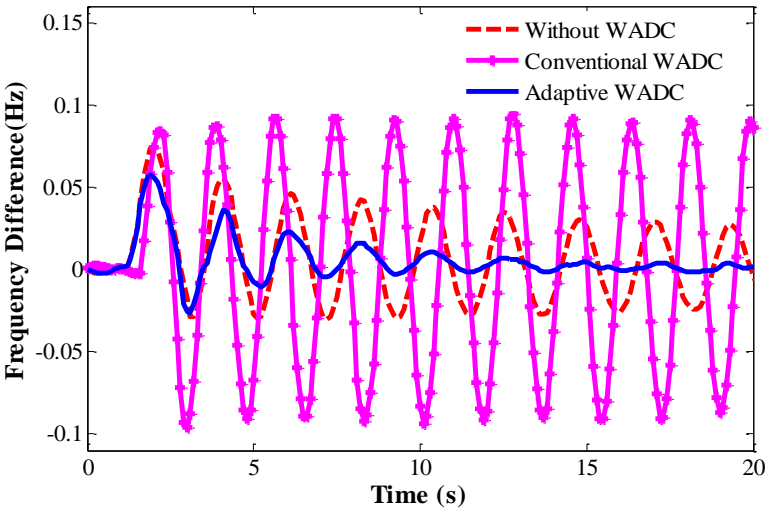

(d) Control performance comparison among of Case 4

Fig.11 Control performances in different cases

The proposed adaptive WADC is compared with the conventional WADC proposed in [3]. Under the operating condition in Case 1 shown in Fig. 11 (a), both the adaptive WADC and traditional WADC can suppress the oscillation. However, when the operating condition is changed from Case 1 to Case 2 or Case 3, the conventional WADC not only cannot suppress the oscillation but also trigger the instable oscillation while the adaptive WADC still has good control performance. From Fig. 11 (d), it can be found that the conventional WADC even become an oscillation source. Note that the conventional WADC is tuned and tested in a similar operating point as Case 1,but the control performance degrades when the operation change, while the adaptive WADC updates the parameters with the identified SISO model that can track the changes of the operation condition.

For Case 1 and 2, the oscillation can be damped in the end even without control actions, but the settled down time was shorten after adaptive WADC added into the system. While Case 3 and Case 4 are poor damping cases, the system will oscillate with a high amplitude without the adaptive WADC. Therefore, the WADC can adapt to a wide range of operating conditions and keep the stability of the power system.

\section{DISCUSSION AND FUTURE WORK}

A phenomenon was found during the experiment. It seems that the WADC can lead to an equal amplitude and higher frequency oscillation compared to the inter-area mode. As shown in Fig.12, there was an event at 1 second. At the same time, the amplitude of the oscillation began to increase. At 10 second, the oscillation came to equal amplitude. If the control system was turned off at 38 second, the amplitude of the oscillation decreased and settled down. If the control 
system was turned on again at 42 second, then the equal amplitude oscillation appeared again. The whole phenomenon indicates that the WADC can become an oscillation source causing the forced oscillation if the parameters of the controller or time delay compensation model are improper for the system. Therefore, it is very important to develop a controller that adapts to a variety operating conditions. The proposed wide-area damping control scheme will be applied to an actual large power system to further demonstrate the effectiveness of the adaptive behavior in the future work. There are some challenges for the actual application as follows: (1) The number of the controllable setpoint signals (generator equipped with PSS) will be a lot. The model will be very complicate if include all the setpoint signals as the model inputs. It is hard to update the model with the operating change online. (2) In a large system, there may be several inter-area modes with poor damping. There may have negative influence among WADCs. (3) The adaptivity of WADC to the sudden operating change of an actual power grid.

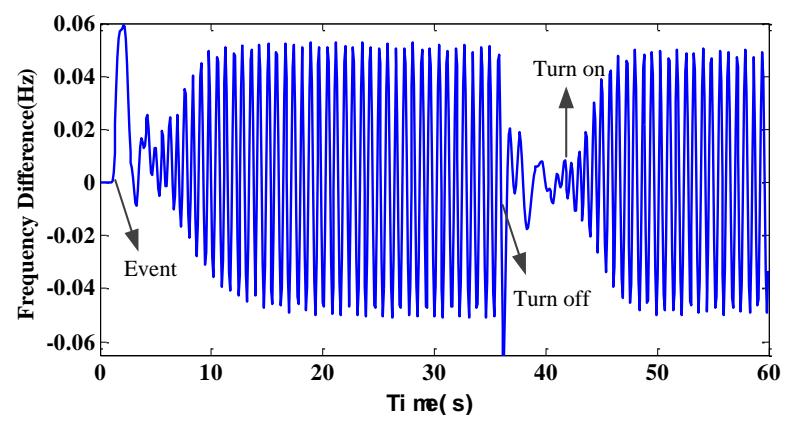

Fig.12 Interesting phenomenon

\section{CONCLUSION}

In this paper, a novel adaptive wide-area damping control system fully based on measurement signals of the power system is proposed and verified. First, FFT analysis and a transfer function identification algorithm was designed to identify the MIMO system model needed to select the best control signal according to the directly obtained residue. Next, a predictive SISO model was identified using the selected best control signal and best feedback signal for predictive control. The SISO model lessened the computational burden and improved the convergence time to meet the requirement of the online implementation. Finally, an adaptive WADC and local time delay compensation system was designed that can update the parameters online with the prediction dominate mode and compensate the time delay locally. On-line implementation on the HTB demonstrates the feasibility of practical realization of a measurement-based wide-area damping control system for small and large disturbances over a wide range of operation conditions.

\section{ACKNOWLEDGMENT}

This work is supported by the Electric Power Research Institute and also makes use of Engineering Research Center Shared Facilities supported by the DOE under NSF Award Number EEC1041877. Additional support is provided by the CURENT Industry Partnership Program. The authors gratefully acknowledge FNET team, Center for ultra-wide-area resilient electric energy transmission networks (CURENT) in the University of Tennessee, US Electric Power Research Institute to support this research.

\section{REFERENCES}

[1] P. Kundur, "Power System Stability and Control," New York, NY, USA: McGraw-Hill, 1994. 
[2] B. Pal, and Balarko Chaudhuri, "Robust control in power systems," Springer, 2005.

[3] M. E. Aboul-Era, A. A. Sallam, J. D. Mccalley, and A. A. Fouad, "Damping controller design for power system oscillations using global signals," IEEE Trans. Power Syst., vol. 11, no. 2, pp. 767-773, 1996.

[4] W. Yao, L. Jiang, J. Wen, Q. H. Wu, and Shijie Chen, "Wide-area damping controller of FACTS devices for interarea oscillations considering communication time delay," IEEE Trans. Power Syst., vol. 29, no. 1, pp. 318-329, 2014.

[5] A. Heniche, I. Kamwa, "Assessment of two methods to select wide-area signals for power system damping control," IEEE Trans. Power Syst., vol. 23, no.2, pp.572-581, 2008.

[6] Y. Zhang, A. Bose, "Design of wide-area damping controllers for interarea oscillations," IEEE Trans. Power Syst., vol. 23, no. 3, pp. 1136-1143, 2008.

[7] W. Masayuki-Vega, H. Takuhei, I. Takanori, M. Yasunori, "Power system stabilization control based on the widearea phasor measurement", Electrical Engineering in Janpan, Vol.163. no.1, pp. 16-24, 2008.

[8] I. Kamwa, G. Trudel, L. Gérin-Lajoie, "Robust design and coordination of multiple damping controllers using nonlinear constrained optimization," IEEE Trans. Power Syst., vol. 15, no. 3, pp. 1084-1092, 2000.

[9] Ch. Zhu, M. Khammash, V. Vittal, W. Qiu, "Robust power system stabilizer design using H $\infty$ loop shaping approach," IEEE Trans. Power Syst., vol. 18, no. 2, pp. 810-818, 2003.

[10]R. Majumder, B.C. Pal, C. Dufour, P. Korba, "Design and real-time implementation of robust FACTS controller for damping inter-area oscillation," IEEE Trans. Power Syst., vol. 21, no. 2, pp. 809-816, 2006.

[11]T. Wang, Z. Wang, J. Liu, J.S. Thorp. Y. Yang, "Classification and regression tree-based adaptive damping control of inter-area oscillations using wide-area signals," IET Generation, Transmission\&Distribution, vol. 8, Iss. 9, pp. 1516$1527,2014$.

[12]M. Zima, M. Larsson, P. Korba, C. Rehtanz, G. Andersson, “ Design aspects for wide-area monitoring and control systems," Pro. of the IEEE, Vol.93,No.5, pp.980-996,2005.

[13]D. K. Chaturvedi and O. P.Malik, "Generalized neuron-based adaptive PSS for multimachine environment," IEEE Trans. Power Syst., vol. 20, no. 1, pp. 358-366, Feb. 2005.

[14]J.B. Zhang, C. Y. Chung, C. Lu, K. Men, and L. Tu, "A novel adaptive wide area PSS based on output-only modal analysis", IEEE Trans. Power Syst., to be published.

[15]W. Yao, L. Jiang, Q. H. Wu, J. Wen, and Shijie Chen, "Delay-dependent stability analysis of the power system with a wide-area damping controller embedded," IEEE Trans. Power Syst., vol. 26, no. 1, pp. 233-240, 2011

[16]H.X. Wu, K.S. Tsakalis, and G.T. Heydt, "Evaluation of time delay effects to wide-area power system stabilizer design, " IEEE Trans. Power Syst., vol. 19, no. 4, pp. 1935-1941, 2004.

[17]K. Tomsovic, D.E. Bakken, V.Venkatasubramanian, and A. Bose, "Designing the next generation of real-time control, communication, and computations for large power systems, "Proc. IEEE, vol. 93, no. 5, pp.965-979, 2005.

[18]I. Kamwa, R. Grondin, and Y. Hebert, "Large-scale active load modulation for angle stability improvement, " IEEE Trans. Power Syst., vol. 14, no. 2, pp. 582-590, 1999.

[19]B. Chaudhuri, R. Majumder, and B.C.Pal, "Wide-area measurement-based stabilizing control of power system considering signal transmission delay," IEEE Trans. Power Syst., vol. 19, no. 4, pp.1971-1979, 2004.

[20]G.J.Li, T.T. Lie, C.B. Soh, G.-H. Yang, "Decentralised nonlinear $\mathrm{H}_{\infty}$ control for stability enhancement in power systems," IET Generation, Transmission\&Distribution, vol. 146, no. 1, pp. 19-24, 1999. 
[21]L. Ljung, System identification: theory for the user 2nd Ed, PTR Prentice, New Jersey, USA, pp1-296, 1999.

[22]N. Zhou, Z. Huang, L. Dosiek, D.Trudnowski, J. W. Pierre, "Electromechanical mode shape estimation based on transfer function identification using PMU measurements," in Proc. of IEEE PES General Meeting, Calgary, Alberta, Canada, July 26-30, 2009.

[23]Y. Liu, K. Sun, Y. Liu, “A Measurement-based Power System Model for Dynamic Response Estimation and Instability Warning”, Electric Power Systems Research, vol. 124, pp.1-9, July 2015.

[24]C. Li, Y. Liu, K. Sun, Y. Liu, N. Bhatt, "Measurement based power system dynamics prediction with multivariate AutoRegressive Model," T\&D Conference and Exposition, 2014 IEEE PES , vol., no., pp.1,5, 14-17 April 2014.

[25]Eriksson, R., X. So, and L. Der, "Wide-area measurement system-based subspace identification for obtaining linear models to centrally coordinate controllable devices," IEEE Trans. Power Del., vol. 26, no. 2, pp. 988-997, 2011.

[26]I. Kamwa, and L. Gerin-lajoie, "State-space system identification-toward MIMO models for modal analysis and optimization of bulk power systems," IEEE Trans. Power Syst., vol. 15, no. 1, pp. 326-335, 2000.

[27]N. Zhou, J. Pierre, and J. F. Hauer, "Initial results in power system identification from injected probing signals using a subspace method," IEEE Trans. Power Syst., vol. 21, no. 3, pp. 1296-1302,2006.

[28]H. Ghasemi, C. Canizares, and A. Moshref, "Oscillatory stability limit prediction using stochastic subspace identification," IEEE Trans. Power Syst., vol. 21, no. 2, pp. 736-745, May 2006.

[29]Sarmadi, S.A.N., and V. Venkatasubramanian, "Electromechanical mode estimation using recursive adaptive stochastic subspace identification," IEEE Trans. Power Syst., vol. 29, no 1, pp. 349-358, Jan. 2014.

[30]N. R. Chaudhuri, A. Domahidi, R. Majumder, B. Chaudhuri, P. Korba S. Ray, and K. Uhlen, "Wide-area power oscillation damping control in Nordic equivalent system," IET Gener. Transm. Distrib., vol. 4, no.10, pp. 1139-1150, Oct., 2010.

[31]W. Yao, L. Jiang, J. Y. Wen, S. J. Cheng and Q. H. Wu, "An adaptive wide-area damping controller based on generalized predictive control and model identification", in Proc. of IEEE PES General Meeting, Calgary, AB, Canada, July 26-30, 2009.

[32]W. Yao, L. Jiang, J. Y. Wen, S. J. Cheng, and Q. H. Wu, "Networked predictive control based wide-area supplementary damping controller of SVC with communication delays compensation," in Proc. of IEEE PES General meeting, Vancouver, BC, Canada, July 21-25, 2013.

[33]W. Yao, L. Jiang, J. Y. Wen, Q. H. Wu and S. J. Cheng,"Wide-area damping controller for power system interarea oscillations: a networked predictive control approach", IEEE Trans. Control Syst. Technology, vol. 23, no. 1, pp. 27$36,2015$.

[34]C. V. Loan, Computational Frameworks for the Fast Fourier Transform SIAM, 1992.

[35]T. Hiyama, N. Suzuki, T. Funakoshi, "On-line identification of power system oscillation modes by using real time FFT, " IEEE Power Engineering Society Winter Meeting, Singapore, 2000.

[36]L. Yang, Y. W. Ma, J.X. Wang, J.Wang, X.H. Zhang, L. M. Tolbert, F. Wang, K. Tomsovic, "Development of coverter based reconfigurable power grid emulator," in Proc. of IEEE ECCE, Pittsburgh, USA, Sep. 13-18, 2014.

[37]M. Klein, G.J. Rogers, P. Kundur, "A fundamental study of inter-area oscillations in power systems," IEEE Trans. Power Syst., vol. 6, no. 3, pp. 914-921, 1991. 